OC6

PEDIATRIC PAIN AT 10 YEARS OLD IN A PORTUGUESE BIRTH COHORT - REPORTED BY CHILDREN

${ }^{1}$ Vanessa Gorito*, ${ }^{2}$ Teresa Monjardino, 1,2,3 Inês Azevedo, ${ }^{2,4}$ Raquel Lucas. 'Department of Pediatrics. Centro Materno Pediátrico, Centro Hospitalar e Universitário de São João, Oporto, Portugal; 'Institute of Public Health of the University of Porto (ISPUP), Oporto, Portugal; ${ }^{3}$ Department of Gynecology-Obstetrics and Pediatrics, Faculty of Medicine of the University of Porto, Oporto, Portugal; ${ }^{4}$ Department of Public Health Sciences and Forensic and Medical Education, Faculty of Medicine of the University of Porto, Oporto, Portugal

\subsection{6/archdischild-2019-epa.6}

Introduction Pediatric pain has been considered an important public health issue which motivates studies about a wide variety of dimensions around the world. Although epidemiological studies have increased over the past years, the information to characterize pediatric pain from population-based studies is still limited, more even when children reported their own pain.

Aim We aim to characterize the prevalence, localization and intensity of pain experience in children of the Generation XXI birth cohort $(\mathrm{G} 21)$ at the age of 10 years, reported by children.

Materials and methods We used data from 4752 ten-year-old children from Generation XXI cohort. Pain was assessed using the Portuguese version of Lubeck Pain Screening Questionnaire applied to children. The statistical significance of differences between groups was assessed using Chi-squared test. Data was analyzed using the SPSS 22.0.

Results Of the 4752 participants, 1668 admitted a pain experience in the previous week (35\%). According to anatomical location, children mentioned more frequently as main pain: upper limb $(n=521)$, abdominal pain $(n=384)$, back pain $(n=384)$, headache $(n=271)$, lower limb $(n=241)$ and chest pain $(n=155)$. These locations differ according to sex $(p<0.001)$. From these children, 309 had pain in two or more different locations in the previous week, with differences between boys and girls $(p=0.035)$. Multsite pain was present in around $4 \%$ of these children. Among these children, more than $66 \%$ of children mentioned moderate pain and more than $26 \%$ admitted intense to severe pain in the last week. We found a statistically difference between the location of principal pain and the intensity valued in the pain face scale $(p<0.001)$. The intensity of pain was also different among boys and girls $(\mathrm{p}=0.005)$.

Discussion This population-based study in Portugal is one of few data available who values children's report of their own pain. At this age, it's normal that parents report pain in place of children. However, literature defends that since preschool, children are able to express their own pain. More than a third of children reported pain in the previous week and about $19 \%$ with 2 or more different anatomical locations. The presence of multisite pain at this age and also the differences found among boys and girls should claim to the attention of investigate the determinants of pain, triggers, impact in children's life's and evolution of pain, namely to chronic pain which is increasing in pediatric age in last years.

\section{OC7 NONSYNDROMIC CRANIOSYNOSTOSIS: REFERRAL PATTERNS FOR METOPIC, CORONAL AND LAMBDOID CRANIOSYNOSTOSIS IN IRELAND; 2009-2018, A 10-YEAR REVIEW}

Luke Trench, Shirley Bracken*, Niall Mclnerney, John Caird, Dylan J Murray. National Paediatric Craniofacial Centre, Temple Street Children's University Hospital, Dublin, Ireland

\subsection{6/archdischild-2019-epa.7}

Background Craniosynostosis is a congenital condition resulting from premature fusion of the cranial sutures resulting in a phenotypic headshape. Nonsyndromic craniosynostosis, involving a single suture only; is the most common presentation involving the fusion of one of the following sutures; sagittal, metopic, coronal or lambdoid. The National Paediatric Craniofacial Centre in Temple Street Children's University Hospital is the designated craniofacial centre in the Republic of Ireland. The treatment of craniosynostosis involves appearance-changing surgery designed to normalise the headshape. As with most other designated craniofacial centres the aim is to perform surgery as close to 12 months of age as possible for these children. The clinical presentation of craniosynostosis as with most rare diseases can result in an over-reliance on different types of radiology imaging, a subsequent later referral and surgery being performed at a later age. The aim of this review was to report the referral patterns of children with metopic, coronal and lambdoid nonsyndromic craniosynostosis presenting to the NPCC.

Methods A retrospective chart review of children who underwent surgery for metopic, coronal or lambdoid craniosynostosis between January 2009 and December 2018 was undertaken. Data collected included: patient demographics, hospital of birth, referrer specialty, date of referral and any radiology investigations performed. Children subsequently diagnosed with a syndrome were excluded.

Results 130 patients were included in the review. 80\% $(n=104)$ were referred before 10 months of age. There was a high use of radiology imaging with $71 \%(n=92)$ of all children having at least one radiology investigation prior to referral. $15 \% \quad(n=19)$ had multiple radiology investigations performed. Paediatricians and Neonatologists referred the largest number of children; 58\% $(n=75)$ and 22\% $(n=28)$ respectively. Surgery was performed before 15 months of age for $44 \%(n=57)$ of children and before 18 months of age for a further 26\% $(n=34)$ of all children.

Conclusion The incidence of metopic, coronal and lambdoid craniosynostosis is in keeping with reported rates in Europe. Although most children were referred before 10 months of age complex referral patterns and use of radiology imaging were associated with later referrals, leading to subsequent delays in surgery performed. It is hoped that an increased awareness of recognising and referring children with craniosynostosis will lead to subsequent treatment as early as possible. The NPCC encourages that referral letters for children with suspected craniosynostosis are accompanied by photographs of the child's headshape and not radiology investigations. 